\title{
La escritura en los niños tarahumaras
}

\author{
Efrén Viramontes Anaya \\ efren8000@hotmail.com \\ Lylia Ana Morales Sifuentes \\ jesusm0124@hotmail.com \\ Luis Manuel Burrola Márquez \\ luisburrola1@yahoo.com.mx
}

\begin{abstract}
Resumen
Esta investigación analiza el desarrollo de la escritura en español de niños indígenas tarahumaras bilingües (del Estado de Chihuahua, México), desde varias perspectivas: la psicogenética, relacionada con el avance en el proceso de construcción del sistema de escritura de los niños investigados; la social, mediante al análisis del contexto familiar, cultural y el entorno del asentamiento de convivencia de los mismos; y la pedagógica, aunque más breve, a través del ambiente áulico dentro de dos escuelas muy diferentes: una regular y otra indígena. El planteamiento central de la investigación giró en torno a la percepción de los múltiples factores que se relacionan con el aprendizaje de la escritura, en un intento de escudriñar analíticamente los elementos posibles de afectación en el proceso referido, así como las implicaciones metodológicas de los maestros, tanto como la dificultad de los mismos para llegar a los alumnos. Un caso en la escuela regular y el segundo en un albergue propio de su etnia. La metodología cualitativa utilizada posibilitó, mediante el estudio de casos, la observación, la entrevista, la videograbación y el análisis de los cuadernos de los niños, percibir situaciones y rescatar evidencias que, mediante el estudio transversal de eventos, personas y contextos, dieron como resultado interpretaciones sobre los factores sociales, culturales, cognitivos y pedagógicos que se percibieron asociados al aprendizaje de la escritura de una lengua que no es la materna, en niños indígenas migrantes establecidos en un medio distinto al de sus ancestros.
\end{abstract}

Palabras clave

Escritura, niños tarahumaras, enseñanza.

\section{Introducción}

Antecedentes y contextualización

El municipio de Rosales es uno de los más pequeños del estado de Chihuahua, se ubica aproximadamente a 81 kilómetros al sur de la Capital del Estado. Su cabecera está localizada al Nor-Oeste de los Municipios de Delicias y Meoqui y en sus inmediaciones está construida la Presa Francisco I. Madero sobre el Río San Pedro.
Como actividades económicas predominan la agricultura y la ganadería, que son una fuente de empleo muy importante para sus habitantes. Su Clima es caluroso la mayor parte del año.

En la periferia de la cabecera se alberga a un asentamiento indígena de tarahumaras desde hace más de 23 años. La problemática que se estudia, relacionada con el aprendizaje de la 
escritura en idioma español por parte de niños tarahumaras establecidos en este municipio, se percibe desde que los indígenas ya asentados, iniciaron con la escolarización de sus hijos en la estructura de la educación formal de la escuela primaria indígena $\mathrm{y}$ las primarias regulares.

Lo que habrá que puntualizar es que el inicio de la investigación se basó en la creencia de que los niños hablaban el tarahumara de manera sistemática como primera lengua, principalmente en sus hogares, no obstante, los padres de los estudiantes analizados, hablan el tarahumara con una frecuencia tal, que paulatinamente éste se va quedando en segundo término de uso con respecto al español.

En los últimos tiempos muchos grupos bajan a los pueblos o ciudades a donde llevan sus artesanías y hierbas medicinales otros más solicitan el Korima que básicamente se refiere a pedir ayuda de cualquier tipo.

En estas migraciones temporales existen familias que se quedan de manera definitiva establecidos en las periferias de las principales poblaciones del estado. Éste es el caso del asentamiento indígena de Rosales Chihuahua, mismo que está integrado por aproximadamente 60 familias.

Los niños tarahumaras que asisten al alberque rural de Rosales, Chih. permanecen en él de lunes a viernes. Los padres de familia los llevan los lunes y los viernes van por ellos.

El personal del alberque lo integran: un director, dos cocineras, dos tutores y un velador. Los tutores de los albergues son personas de formación pedagógica, generalmente ingresan con estudios de bachillerato y les basifican la plaza hasta que concluyen los estudios de licenciatura en educación.

El albergue tiene capacidad para asistir a 50 niños y en la actualidad se atiende a 46, de preferencia de lugares de donde no existe escuela primaria o de familias de muy bajos recursos económicos, 26 de ellos son tarahumaras. La función primordial es de dar servicios asistenciales de hospedaje, alimentación y aseo personal, además de dar apoyos académicos y formativos diversos. Con estos servicios se atiende de manera preventiva la posibilidad del fracaso escolar.

Los niños que asisten a las escuelas primarias y el albergue no conocen la sierra Tarahumara, ya son niños nacidos en esta región. Por lo tanto, están perdiendo su cultura y están asumiendo la de la región. Muy pocos de ellos hablan ya el tarahumara.

Los niños ingresan a la escuela primaria generalmente a los 7 u 8 años. En promedio lo hacen un año después que la generalidad de los niños de la escuela primaria regular. Algunos no concluyen sus estudios en el tiempo establecido para hacerlo: 6 años.

\section{Problema de investigación}

El análisis de las dificultades que tienen los niños tarahumaras en su aprendizaje y los docentes en la enseñanza del sistema de escritura, es un tema de estudio que cobra importancia en el sentido de aportar una explicación, a partir de estudios de caso, de qué es lo que sucede en dicho proceso cuando los que aprenden (los niños) no pertenecen al contexto sociocultural ni lingüístico del lugar en que lo hacen.

También tiene una importancia particular el hecho de observar las dificultades que los docentes tienen al atender un grupo regular y a otro multigrado de niños tarahumaras en dos 
escuelas totalmente diferentes en una misma localidad semirural.

El planteamiento principal gira en torno a las dificultades que tienen los alumnos tarahumaras, de ambas escuelas, en el aprendizaje de la escritura en español. Los ámbitos a considerar son: el trabajo de los niños y de los docentes, además del contexto familiar y social del asentamiento indígena.

La realización de esta investigación aporta niveles de comprensión de las causas por las cuales existen esas dificultades en los docentes y en los estudiantes tarahumaras al momento de construir, desde cada escuela, el sistema de escritura del español.

El análisis de la construcción del sistema de escritura por parte de los niños tarahumaras se realizó a partir de la aportación de un esquema explicativo, a la luz de los avances teóricos que existen sobre el tema de estudio, enriquecidos por los elementos contextuales que fortalecen o contradicen dichas teorías.

Los resultados de esta investigación explican de manera concreta lo que sucedió en las escuelas Benito Juárez y Rarajípame de Rosales, Chih., con respecto al proceso de enseñanza y aprendizaje de la escritura en los grupos de primer grado de esas escuelas y éstos pueden ser extrapolados a otros contextos como un modelo explicativo de situaciones similares.

Derivado de las precisiones anteriores el problema de investigación se plantea de manera concreta como sigue: ¿Qué factores se asocian al aprendizaje de la escritura de los niños tarahumaras del asentamiento de Rosales, Chih.?

\section{Propósitos}

Analizar el proceso de la construcción de la lengua escrita de los niños tarahumaras (primer ciclo de la educación primaria).
Conocer las principales dificultades de los niños y los docentes en el proceso de construcción de la escritura.

Conocer las aportaciones o limitaciones del contexto familiar, cultural y social para el aprendizaje de la escritura. Generar elementos de insumo para propuestas aplicables a casos similares.

\section{Enfoque y fundamentación teórica del objeto de investigación}

Para efectos de realizar un análisis e interpretación del proceso que siguen los niños Tarahumaras en la construcción de la escritura en el idioma español se revisaron dos especialistas, principalmente Gómez Palacio et. al. (2000) y Nemirovsky (2004). Con relación a las características de las lenguas indígenas se incluyen las aportaciones de Vigil (2010), de Silvia Schmelkes (2002) en lo relacionado con las ideas de multiculturalidad, pluriculturalidad e interculturalidad y de Echeverría González (2001) respecto a los problemas educativos de los tarahumaras.

\section{Metodología Método de casos}

La metodología utilizada para esta investigación es de tipo cualitativa, a través de lo que se establece como estudio de método de casos, porque se analiza una situación detalladamente, proporcionando una teoría naturalista de la situación.

El estudio de caso es explicado por Martínez (2005) y Stake (2007), de los cuales se considera el planteamiento metodológico para esta parte de la investigación.

Los 4 casos en esta investigación están representados por 3 niños tarahumaras del grupo de primer grado de la escuela primaria indígena Rarajípame: Rosario, Luna y Lorenzo y por Natalia de la escuela primaria regular Benito Juárez, de 
Rosales, Chih.

\section{La observación}

En la investigación la observación se realizó en 16 sesiones de primer grado, en la asignatura de español, relacionadas con la enseñanza y aprendizaje de la lengua escrita. Se focalizó a las acciones que realizan los niños tarahumaras y las actividades didácticas o de intervención pedagógica que realiza el docente en la enseñanza de la escritura. Los datos que se obtuvieron aquí son evidencias de avance de construcción del sistema de escritura en los niños, las actividades didácticas del docente, las dificultades de los alumnos y maestros, así como elementos del contexto familiar social de los niños implicados.

También fue motivo de observación el contexto socioeconómico, familiar y cultural en el que se desenvuelven los alumnos tarahumaras. Los datos obtenidos son características, descripciones y evidencias de la influencia del contexto en la formación de los alumnos.

\section{La entrevista}

En el proceso de la investigación se realizaron once entrevistas:

Cuatro para niños tarahumaras, para encontrar sentido e información sobre el proceso de construcción de la lectura.

Cuatro a padres de familia, para obtener información del contexto, las actividades familiares y de apoyo a la educación de los niños, así como de otros problemas o asuntos relativos con el desarrollo del sistema de escritura de sus hijos.

Dos entrevistas a los maestros de los grupos de primer grado de la escuela primaria, con el propósito de indagar sobre las dificultades de enseñanza, de aprendizaje y de las acciones concretas que realizan para sortear dichas dificultades.

La onceava se efectuó al encargado del albergue de Rosales, Chih., en el que se encuentran asistidos los niños tarahumaras del asentamiento indígena.

Con los docentes de ambas escuelas se pretendió saber cómo era el clima de trabajo en el aula, la participación de los niños en las actividades escolares (principalmente los tarahumaras), los avances académicos observados, los apoyos específicos, los procesos metodológicos realizados y además sobre la habilitación profesional para desempeñarse en sus funciones.

Con respecto al director del albergue el propósito fue indagar sobre los apoyos que brinda la institución, cómo perciben el avance académico de los niños que atiende, específicamente en el desarrollo de la escritura, y el perfil profesional del personal que labora en la institución.

También se realizaron análisis de los trabajos y cuadernos de los niños, para observar el avance y dificultades que tienen en la construcción del sistema de escritura del español. Eventualmente, junto con el análisis de los cuadernos y trabajos se efectuó una entrevista clínica a los niños para comprender los procesos que siguen en la construcción de la escritura.

\section{Resultados}

Avance de la escritura de los casos y de la generalidad de los niños tarahumaras El proceso de construcción del sistema de escritura, no sólo del Estado de Chihuahua, sino también de México, se desarrolla de manera más sistemática durante el primer grado de la escuela primaria. En la generalidad de los niños, llegan a 
construirlo en el nivel alfabético entre los 6 y 7 años de edad. Sin embargo, los niños migrantes tarahumaras, además de ingresar a la educación formal de la escuela primaria entre los 7 y 8 años, se tardan en promedio dos años para lograr desarrollar el sistema en ese mismo nivel. Es común que se observe en los asentamientos indígenas que los niños tarahumaras acceden al nivel alfabético entre los 8 y 9 años de edad, dos años más tarde que los niños chabochis.

Los niveles de construcción del sistema de escritura del español en los niños analizados en los grupos de ambas escuelas se muestran en la tabla 1 :

Tabla 1: Porcentaje de niños por niveles de escritura

\begin{tabular}{|c|c|c|c|c|c|c|c|c|c|}
\hline \multirow{2}{*}{ Escuela } & \multirow{2}{*}{$\begin{array}{c}\text { No. } \\
\text { de } \\
\text { alum } \\
\text { nos }\end{array}$} & \multicolumn{2}{|c|}{ Nivel alfabético } & \multicolumn{2}{|c|}{$\begin{array}{l}\text { Nivel silábico } \\
\text { alfabético }\end{array}$} & \multicolumn{2}{|c|}{ Nivel Silábico } & \multicolumn{2}{|c|}{ Nivel Presilábico } \\
\hline & & $\begin{array}{l}\text { Absolu } \\
\text { to }\end{array}$ & $\begin{array}{c}\text { Porcent } \\
\text { aje }\end{array}$ & $\begin{array}{c}\text { Absolu } \\
\text { to }\end{array}$ & $\begin{array}{c}\text { Porcent } \\
\text { aje }\end{array}$ & $\begin{array}{c}\text { Absolu } \\
\text { to }\end{array}$ & $\begin{array}{c}\text { Porcent } \\
\text { aje }\end{array}$ & $\begin{array}{c}\text { Absolu } \\
\text { to }\end{array}$ & $\begin{array}{c}\text { Porcent } \\
\text { aje }\end{array}$ \\
\hline Regular & 24 & 21 & 87.5 & 1 & 4.16 & 2 & 8.33 & 0 & 0 \\
\hline $\begin{array}{l}\text { Indígen } \\
\text { a }\end{array}$ & 12 & 0 & 0 & 1 & 8.33 & 5 & 41.66 & 6 & 50 \\
\hline
\end{tabular}

El problema del aprendizaje de la escritura en una lengua que no es la materna, reside principalmente en que se tienen pocos referentes del contexto familiar y social que sirvan de base y soporte para la construcción del sistema de escritura.

El contexto familiar y social de los niños tarahumaras es un factor importante en el aprendizaje de la escritura del español como segundo idioma.

El ambiente de interacción social del grupo escolar genera oportunidades específicas de aprendizaje que son subutilizadas por el maestro de la escuela indígena.

Expectativas académicas de los tarahumaras es muy baja, en lo general no va más allá del nivel primario, toda vez que para la edad de cursar la secundaria es cuando regularmente se casan o se juntan las parejas en vida conyugal.

El aprovechamiento de la interacción social en el grupo por parte del maestro, facilita el aprendizaje de la escritura. Esta situación no es aprovechada por el maestro de la escuela indígena.

La participación del albergue en el desarrollo académico de los tarahumaras a través del apoyo tutorial escolar, servicios asistenciales, actividades deportivas $\mathrm{y}$ culturales.

Problemas de enseñanza de los docentes que atienden a los niños tarahumaras son más pronunciados que los de la escuela regular. Tienen menos apoyos técnico pedagógico y los niveles de preparación académica son más bajos.

La exigencia académica de la escuela primaria regular es mucho más alta que la que se percibe en la escuela primaria indígena. Aun cuando en esta última se tengan apoyos de computadora con enciclomedia, impresora, libros, materiales diversos de la SEP, aire acondicionado en el salón de clases.

Los libros editados especialmente 
para la etnia Tarahumara tienen la desventaja de que muchas de las palabras no las entienden los niños por las variedades lingüísticas que existen en las regiones de la sierra Tarahumara, de donde provienen sus progenitores. Esto coincide con las conclusiones que al respecto tiene Schmelkes (2002), cuando señala que: "Entre las comunidades hay pequeñas variaciones lingüísticas, tanto de pronunciación como de vocabulario. La identidad comunitaria, vinculada a la lengua, conduce a rechazar como propia una lengua que [...] difiere ligeramente de la forma utilizada en la comunidad propia" (p. 12). Por lo tanto, es importante establecer que existe concordancia con esta misma especialista, con respecto a que los libros de texto que se utilizan en las comunidades indígenas tienen limitaciones de identidad lingüística en el uso estandarizado con todos los grupos tarahumaras.

\section{Conclusiones}

En el trabajo realizado en esta investigación se pudieron identificar algunos aspectos que son necesarios considerar al momento de trabajar con grupos donde haya niños con características similares a los casos estudiados.

En la mayoría de los tarahumaras se observa un problema de sintaxis en el lenguaje oral. Generalmente no coinciden con la convencionalidad los usos que hacen de género y número.

El avance del aprendizaje del sistema de escritura es más lento en los niños indígenas que en los mestizos, mientras que estos últimos lo aprenden en promedio entre los 6 y 7 años, los tarahumaras lo hacen entre los 7 y 9, con mucho más dificultad.

El clima de trabajo en la escuela regular es más exigente que en la escuela indígena; sin embargo, la interacción entre pares se ve más favorecida la escuela indígena, por lo menos para los niños tarahumaras.

El ambiente familiar es un factor determinante para el ingreso, permanencia y aprendizaje en la escuela primaria. En lo general el ambiente de las familias de los mestizos es más alfabetizador que el de los tarahumaras.

El contexto general del asentamiento indígena en lo general es poco alfabetizador. Las calles por las que transitan habitualmente los niños desde el albergue hasta la escuela primaria y los alrededores de su casa, carecen de portadores de textos que le permita considerar la escritura como parte del medio en el que vive.

Los maestros de las escuelas regular e indígena tienen diferencias en las exigencias académicas que caracterizan las actividades de enseñanza. La escuela regular es más exigente que la indígena.

Se percibe en los trabajos y actividades de los niños que existe un enfoque metodológico más favorecedor en la escuela regular. Éste es un problema de enseñanza que se observa más en la escuela indígena.

Los problemas de aprendizaje de los niños tarahumaras están determinados por los factores familiares, contextuales, culturales y académicos de sus escuelas y maestros.

Los libros de texto específicos para cada etnia indígena tienen limitaciones de identidad lingüística considerables, debido a la amplia variedad de expresiones lingüísticas regionales.

La condición de aprender a escribir en una lengua o idioma que no es el materno genera divergencias entre los especialistas. Sin embargo, se pudo 
observar y constatar con los docentes indígenas que es más fácil iniciar con la enseñanza de la escritura en español, más que en su lengua indígena, toda vez que esta última es una lengua ágrafa.

Las dificultades en el aprendizaje de los niños tarahumaras, migrantes establecidos, se perciben como determinadas por los factores familiares, contextuales, culturales y académicos de sus escuelas y maestros.

El principio que se propone para que la educación de los indígenas migrantes sea más relevante y pertinente, en los términos establecidos en las políticas educativas actuales de México, es la promoción efectiva de la educación intercultural (Schmelkes (2002 y 2005), toda vez que los niños se enfrentan con una cultura propia a un proceso de aprendizaje que pertenece a otra cultura (mestiza), misma que no debe ser desdeñada tampoco porque es a la que se están integrando como ciudadanos de una comunidad más amplia que la de su asentamiento o barrio. En este sentido, tendrán mejores oportunidades de participar en los eventos ciudadanos, con derechos y obligaciones, así como en la exigencia de promover las compensaciones de atención generadas por la relegación histórica de la que han sido objeto, respetando el fomento de sus costumbres y tradiciones.

\section{Referencias}

Echeverría G, H. (2001). La educación indígena en Chihuahua; hacia la construcción de la interculturalidad. En H. Echeverría (Coord.), Antología asignatura regional I (pp. 42-46). Chihuahua, México: Secretaría de Educación y
Cultura SEP.

Ferreiro, E y Teberosky, A. (2007). Los sistemas de escritura en el desarrollo del niño. 23 ed. México. Siglo xxi editores.

Gómez P, M. (compilador) (1991). Nuevas perspectivas sobre los procesos de lectura y escritura. Edición 8a ed. Publicación Buenos Aires: Siglo Veintiuno.

Hernández S, R., Fernández C, C. y Baptista L, P. (2006). Metodología de la investigación. Edición 4a.Ed. McGraw-Hill Interamericana.

Martínez, M. (2005), Actualización de la epistemología y metodología en educación, III Jornada de Innovación Educativa. Recuperado el 29 de abril del 2009 en http://www.ucla.edu.ve/viacadem /redine/jornadas/III\%20Jornadas \%20REDINE/Ponencia sdelasIIIJornadas.htm

Nemirovsky, M. (2004). Coord Antes de empezar: ¿Qué hipótesis tienen los niños acerca del sistema de escritura? SEP, México.

Schmelkes, S. (2002). La enseñanza de la lectura y la escritura en contextos multiculturales Conferencia presentada en el VII Congreso Latinoamericano para el Desarrollo de la Lectura y la Escritura, celebrado en Puebla, Pue., del 16 al 19 de octubre de 2002. www.amdh.com.mx/ocpi/docume ntos/docs/6/15.doc

Stake, R., (2007). Investigación con estudio de casos. Madrid, España: Morata.

Vigil, N. Pueblos indígenas y escritura. http://interculturalidad.org/nume ro03/2_07.htm. (Recuperado 20 de febrero de 2010) 
RECIE. Revista Electrónica Científica de Investigación Educativa

Vol. 1, núm. 1, enero-diciembre 2012, pp. 149-155.

Viramontes Anaya, E.; Morales Sifuentes, L.A.; y Burrola Márquez, L.M. 